\title{
Physician-Driven Discretionary Utilization: Measuring Overuse and Choosing Wisely
}

\author{
Gregory W Ruhnke, MD, MS, MPH, ${ }^{1 *}$ R Sacha Bhatia, MD, MBA ${ }^{2,3}$
}

${ }^{1}$ Section of Hospital Medicine, Department of Medicine, University of Chicago, Illinois; ${ }^{2}$ Institute for Health System Solutions and Virtual Care, Women's College Hospital, Toronto, Ontario, Canada; ${ }^{3}$ Institute of Health Policy, Management and Evaluation, University of Toronto, Canada.

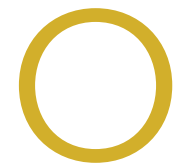

verutilization and low-value care are important clinical and policy problems. Their measurement is challenging because it requires detailed clinical information. Additionally, there are inherent difficulties in identifying discretionary services likely to be inappropriate or low-value and demonstrating that certain services produce little/no health benefit. Quantifying "ideal" expected testing rates-ones that would reflect minimization of inappropriate/low-value care without excluding essential, high-yield diagnostic services_-presents additional challenges. Consequently, of 521 unique measures specified by national measurement programs and professional guidelines, $91.6 \%$ targeted underuse, while only $6.5 \%$ targeted overuse. ${ }^{1}$

The potential for unintended consequences of implementing measures to eliminate overuse are a barrier to incorporating such measures into practice. ${ }^{2}$ For example, measuring, reporting, and penalizing overuse of inappropriate bone scanning may lead to underuse in patients for whom scanning is crucial. ${ }^{2}$ Most overuse measures based on inappropriate or low-value indications relate to imaging and medications. ${ }^{1}$ However, there is increasing interest in overutilization measures based on a broad set of health services. Identifying low-value testing or treatments often requires a substantial degree of clinical detail to avoid the damaging inclusion of beneficial services, which may lead to unintended negative outcomes, creating skepticism among clinicians. Ultimately, getting measurement of low-value care wrong would undermine adoption of interventions to reduce overuse.

To reduce low-value care through expansive measures of provider ordering behavior, ${ }^{3}$ Ellenbogen et a $\left.\right|^{4}$ derived a novel index to identify hospitals with high rates of low-yield diagnostic testing. This index is based on the concept that, in the presence of nonspecific, symptom-based principal diagnoses, a substantial proportion of (apparently) non-diagnostic related studies were probably ordered despite a low pretest probability of serious disease. Since such symptom-based diagnoses reflect the absence of a more specific diagnosis, the examinations observed are markers of physician-driven decisions leading to discretionary utilization likely to be of low-value to patients. This study fills a critical gap in dual measures of appropriateness and yield, rather than simply utilization, to advance the Choosing Wisely campaign. ${ }^{3}$

*Corresponding Author: Gregory W Ruhnke, MD, MS, MPH; Email: gruhnke@ medicine.bsd.uchicago.edu; Telephone: 773-834-8350.

Received: October 26, 2020; Revised: October 28, 2020;

Accepted: Accepted October 28, 2020

๑ 2021Society of Hospital Medicine DOI 10.12788/jhm.3559
Advantages of this overuse index include its derivation from administrative data, obviating the need for electronic health records, and incorporation of diagnostic yield at the inpatient-encounter level. One study selected procedures identifiable solely with claims from a set deemed overused by professional/ consumer groups. ${ }^{5}$ However, the yield of physician decisions in specific cases was not measured. In contrast, this novel index is derived from an assessment of diagnostic yield. ${ }^{4}$ Although test results are not known with certainty, the absence of a specific discharge diagnosis serves as a test result proxy. Measurement of diagnostic examination yield at the patient-level (aggregated to the hospital-level) may be applicable across hospitals with varied patient populations, which include large differences in patient and/or family preferences to seek medical attention and engage in shared decision-making. The role that patient preferences play in decisions creates a limitation in this index-while decisions for the candidate diagnostic tests are physician driven, patient demand may be a confounding factor. This index cannot therefore be considered purely a measure of physician-induced intensity of diagnostic services. Patient-reported data would enhance future analyses by more fully capturing all dimensions of care necessary to identify low-value services. Subjective outcomes are critical in completely measuring the aggregate benefits of tests and interventions judged low-value based on objective metrics. Such data would also aid in quantifying the relative contributions of patient and physician preferences in driving discretionary utilization.

Finally, the derived index is restricted to diagnostic decision-making and may not be applicable to treatment-related practice patterns. However, the literature suggests strong correlations between diagnostic and therapeutic intensity. Application of this novel index will play an important role in reducing low-value discretionary utilization.

Disclosures: The authors have nothing to disclose.

\section{References}

1. Newton EH, Zazzera EA, Van Moorsel G, Sirovich BE. Undermeasuring overuse--an examination of national clinical performance measures. JAMA Intern Med. 2015;175(10):1709-1711. https://doi.org/10.1001/jamainternmed.2015.4025

2. Mathias JS, Baker DW. Developing quality measures to address overuse. JAMA. 2013;309(18):1897-1898. https://doi.org/10.1001/jama.2013.3588

3. Bhatia RS, Levinson W, Shortt $S$, et al. Measuring the effect of Choosing Wisely: an integrated framework to assess campaign impact on low-value care. BMJ Qual Saf. 2015;24(8):523-531. https://doi.org/10.1136/bmjqs-2015-004070

4. Ellenbogen MI, Prichett L, Johnson PT, Brotman DJ. Development of a simple index to measure overuse of diagnostic testing at the hospital level using administrative data. J Hosp Med. 2021;16:xxx-xxx. https://doi.org/10.12788/jhm.3547

5. Segal JB, Bridges JF, Chang HY, et al. Identifying possible indicators of systematic overuse of health care procedures with claims data. Med Care. 2014;52(2):157-163. https://doi.org/10.1097/MLR.0000000000000052 